\title{
Maternal weight and lean body mass may influence the lactation-related bone changes in young undernourished Indian women
}

\author{
Bharati Kulkarni*, Veena Shatrugna, Balakrishna Nagalla, P. Ajeya Kumar, K. Usha Rani \\ and A. Chandrakala Omkar \\ National Institute of Nutrition (Indian Council of Medical Research), Jamai Osmania PO, Hyderabad 500 007, India \\ (Received 8 February 2008 - Revised 7 July 2008 - Accepted 2 September 2008 - First published online 30 October 2008)
}

Lactation is known to be associated with a transient loss of bone mineral density (BMD) during 3-6 months post-partum. Bone changes during lactation in women consuming low dietary calcium are not sufficiently studied. The present longitudinal study examined the BMD changes during lactation in undernourished women and the relationship of bone changes to the nutritional status. Whole-body bone mineral content and BMD at hip, lumbar spine and forearm were assessed using dual-energy X-ray absorptiometry in thirty-six lactating women from the low socio-economic group at four time points - within 1 month after delivery (baseline), and at 6,12 and 18 months after delivery. Maternal body composition and biochemical parameters of bone metabolism were estimated at the same time. It was observed that femoral neck BMD reduced by $4.6 \%$ at 6 months, but recovery to the baseline was incomplete at 18 months with a deficit of $2 \%$. Hip BMD reduction at 6 months was transient. Lumbar spine BMD did not show significant loss at 6 months and BMD increased by 3.6 and $6.3 \%$ at 12 and 18 months, respectively. Regression analyses indicated that baseline lean mass was the most important determinant of bone preservation at femoral neck, hip as well as whole body, whereas baseline body weight was the most important determinant of per cent gain in lumbar spine. Maternal nutritional status as indicated by body weight and lean mass appears to influence the lactation-related BMD changes in undernourished women from the low socio-economic group in India.

Bone mineral density: Lactation: Nutrition: Lean body mass: Women: BMI

A breast-feeding mother secretes about 200-300 mg of calcium into breast milk every day and compensatory mechanisms to conserve calcium such as reduced urinary calcium excretion and increased maternal bone mineral turnover are active during lactation ${ }^{(1-3)}$. Studies from the west have shown a 4-7\% significant reduction of maternal bone mineral density (BMD) at lumbar spine and hip regions during 3-6 months of lactation $^{(4-6)}$. Women who breast-fed for a longer time had a higher bone loss ${ }^{(7)}$. Restoration of bone mineral density is known to occur following resumption of menstruation ${ }^{(2,4,7-9)}$.

The afore-mentioned studies have been reported from developed countries, where women have moderate to high dietary intakes of calcium. The consequences of low calcium intake during lactation are not sufficiently studied. It is possible that magnitude and determinants of lactation-related bone changes are different in undernourished women consuming low calcium in their diets when compared with the well-nourished women consuming diets rich in calcium. A study in lactating Brazilian women with calcium intakes of about $460 \mathrm{mg} / \mathrm{d}$ suggested that the net balance in bone calcium turnover was positively associated with dietary calcium intake ${ }^{(10)}$, while other Western studies in women consuming over $800 \mathrm{mg}$ of calcium per day have indicated that bone changes during lactation were not influenced by maternal dietary calcium intakes ${ }^{(11,12)}$.

Indian women from low socio-economic group subsisting on cereal-based low-calcium diets ${ }^{(13)}$ breast-feed their infants for a prolonged period of time, often until 2 years of age. In addition, their calcium requirements during pregnancy and lactation remain high because of childbearing at a young age, probably before attainment of peak bone mass (PBM).

Studies from India have demonstrated the poor bone health of women from low socio-economic group as indicated by early fractures and a high prevalence of osteoporosis as early as 40 years of age $\mathrm{a}^{(14-16)}$. Prolonged lactation with poor dietary intake of calcium and other nutrients in these women might strain the calcium balance and contribute to bone thinning and early osteoporosis. A descriptive longitudinal study was therefore planned to map the BMD changes during lactation using a sensitive technique of dual-energy X-ray absorptiometry.

\section{Subjects and methods}

Sample size

Assuming $95 \% \mathrm{CI}$ and $80 \%$ power, standard deviation of BMD at lumbar spine $-0.1 \mathrm{~g} / \mathrm{cm}^{2}$, correlation coefficient of spine BMD at two time points -0.91 and expected difference in spine BMD of $2 \%$, the required sample size was calculated to be thirty-four. Assuming $20 \%$ loss to follow-up, it was decided to enrol forty women.

Abbreviations: BA, bone area; BMD, bone mineral density; BMC, bone mineral content; LS-BMB, lumbar spine bone mineral density; PBM, peak bone mass 


\section{Subjects}

Forty consecutive women who attended the post-partum follow-up clinic in a large Government Maternity Hospital (Hyderabad, India) were enrolled for the afore-mentioned study during the period of June-September 2002. All the women had established breast-feeding, had undergone tubectomy and visited the clinic for their first follow-up within 1 month post-partum. Since only women who had undergone tubectomy were enrolled in the study, there were no women with parity 1 . Parity of the study group women ranged from 2 to 5 . None of the women were consuming medications that are known to affect bone metabolism. The study was approved by the institutional ethics committee. Informed consent was obtained from all the subjects.

\section{Study design}

Subjects were examined at four time points during the study period - within 1 month after delivery (baseline), and at 6, 12 and 18 months after delivery. At each time point, the following were assessed - maternal anthropometry (height at baseline and weight), infant's weight and length, maternal bone parameters at hip, lumbar spine, forearm and whole body, maternal body composition including lean mass, fat mass and per cent fat. Blood was drawn for estimation of biochemical parameters at each time point. Dietary intakes were assessed at two time points in a subsample of $50 \%$ women once during the 3-6 months post-partum and second time - at about 12 months post-partum. Information regarding resumption of menstruation and introduction of complementary foods was collected at each time point.

Customary medical care and nutrition advice were provided to all the subjects. The women breast-fed as long as they wished. They were advised to start energy-dense complementary foods at infant's 6 months of age as per the WHO guidelines.

\section{Procedures}

Anthropometry. Maternal weight was measured without footwear to the nearest $0.1 \mathrm{~kg}$ on lever-type SECA balance (Hamburg, Germany). Heights were measured to the nearest $0.1 \mathrm{~cm}$ with a stadiometer (SECA, Birmingham, UK). Infant's weight was measured with lever-type SECA mechanical baby beam balance (Hamburg, Germany), and length was measured with an infantometer made in-house at our Institute. Quality control checks were carried out every 3 months.

Bone mineral density measurements. BMD measurements were carried out using dual-energy X-ray absorptiometry (Hologic QDR $4500 \mathrm{~W}$, Waltham MA, USA) at anteroposterior lumbar spine (L1-L4), hip and forearm as well as whole body including body composition. All scans and analyses were carried out according to the manufacturer's instructions by a trained technician. The scanner was calibrated daily and its performance was monitored as per the quality assurance protocol. No sign of scanner drift was observed during the study period.

The in vivo precision (CV \%) was $1 \%$ for lumbar spine and hip BMD and $<1 \%$ for whole-body bone mineral content (BMC) measurements. Manufacturer's normative data were used as a reference range.
Biochemical parameters. A fasting blood sample was drawn in the morning between 09.00 and 10.00 hours in all the subjects and the estimations of biochemical parameters were carried out using standard procedures. $\mathrm{Hb}$ was estimated by the cyanmethaemoglobin method. Serum albumin ${ }^{(17)}$, serum tartrate-resistant acid phosphatase ${ }^{(18)}$ and serum bonespecific alkaline phosphatase ${ }^{(19)}$ estimations were carried out on the same day.

The serum was processed and preserved, and estimation of serum calcium was done within a week of sample collection using atomic absorption spectroscopy. Breast milk sample (1-2 ml) was expressed manually into a sterile tube and calcium levels were estimated using atomic absorption spectroscopy.

Dietary calcium intakes. Dietary calcium intakes were estimated by $24 \mathrm{~h}$ recall method by a trained nutritionist. The nutrient intakes were determined using published food composition data ${ }^{(20)}$.

\section{Statistical analyses}

SPSS Windows version 14.0 was used for statistical analysis. Descriptive statistics were calculated for all the bone parameters at different sites and time points, anthropometric measurements of mother and offspring, nutrient intakes and demographic variables like age and parity. Prevalences were calculated at different points for biochemical parameters. $Z$ scores of height for age and weight for age were also calculated. Mean values of bone parameters at different time points were compared by repeated-measures ANOVA with post hoc test for each site. Per cent change from baseline was calculated as ((final value - baseline value $) \times 100) /($ baseline value) for each bone parameter and site. Regression models were built to study the relationship of per cent change in BMD at different skeletal sites as dependent variables and height, weight, age, parity, lean mass, fat mass, time of baseline measurements since delivery and duration of post-partum amenorrhoea as independent variables.

\section{Results}

A total of forty women were enrolled for the study. However, thirty-six women completed follow-up till 18 months. The reasons for loss to follow-up were a change of residence (in two) and cessation of breast-feeding consequent to child's illness (in two). Characteristics of the women who were lost to follow-up were not different from those of the study women. Some women had metal jewellery that interfered with the scan area and thus the BMD measurements. The jewellery added about $1 \mathrm{~g}$ of mineral and therefore such scans were not included in the study. Therefore, whole-body measurements were available in thirty-two, lumbar spine measurements in thirty-five and forearm measurements in twenty-eight women. Measurements at the hip were available in all the women. The women enrolled in the study lived in urban slums and belonged to the low socio-economic group. All of them had singleton full-term deliveries. Twenty-eight women had vaginal deliveries, where as eight had Caesarean sections. The characteristics of the subjects are presented in Table 1. It is important to note that these women had their first child at 19 years of age, completed childbearing at 23 years and had a tubectomy done after the birth of the index child (parity of 2.6). Maternal height, weight and 
Table 1. Baseline characteristics of the study women ( $n$ 36)

(Mean values with their standard errors)

\begin{tabular}{lrc}
\hline & Mean & SE \\
\hline Age (years) & 23.5 & 0.5 \\
Age at first pregnancy (years) & 19.0 & 0.4 \\
Parity & 2.6 & 0.1 \\
Height $(\mathrm{cm})$ & 150.7 & 0.8 \\
Weight $(\mathrm{kg})$ & 45.5 & 0.9 \\
BMI $\left(\mathrm{Kg} / \mathrm{m}^{2}\right)$ & 20.0 & 0.4 \\
Birth weight $(\mathrm{kg})$ & 2.8 & 0.6 \\
Duration of post-partum amenorrhoea (months) & 7.0 & 0.8 \\
Time since delivery at baseline scan (d) & 16.0 & 0.9 \\
\hline
\end{tabular}

birth weight of the babies were comparable to the data from the low socio-economic group from India and much lower when compared with well-nourished women and infants from the west. The mean duration of post-partum amenorrhoea was 7 months with a wide variation.

\section{Dietary intakes}

Dietary intakes are presented in Table 2. There were no significant differences in the nutrient intakes during early and later part of lactation and therefore, pooled values are given in Table 2. Diets were typically cereal based with very low intake of protective foods such as milk and milk products, flesh foods, fish, fruits and vegetables. Intake of all the major nutrients was below the RDA $^{(21)}$ for lactating women.

\section{Duration of breast-feeding}

All the women except three breast-fed their infants throughout the study period of 18 months. One stopped breast-feeding after 6 months and two stopped after 1 year. Five women practised exclusive breast-feeding during the first 6 months postpartum. Commonly, small sips of water and animal milk feeds were offered during first 6 months, and semi-solid and solid foods were offered in the second half of infancy.

\section{Changes in bone and body composition parameters during} lactation

The baseline values of bone and body composition parameters and changes at four time points during the post-partum period

Table 2. Dietary intakes of the study subjects $(n 18)$

(Mean values with their standard errors)

\begin{tabular}{lccr}
\hline & \multicolumn{2}{c}{ Intakes } \\
\cline { 2 - 3 } Nutrients & Mean & SE & RDA \\
\hline Energy (kj) & 7908 & 264 & 11610 \\
Energy (kcal) & 1890 & 63 & 2775 \\
Proteins (g) & 43.3 & 1.7 & 75 \\
Fat (g) & 39.0 & 3.1 & 45 \\
Calcium (mg) & 449 & 31 & 1000 \\
Phosphorus (mg) & 944 & 36 & \\
Vitamin A (retinol) ( $\mu \mathrm{g})$ & 286 & 33 & 950 \\
Thiamine (mg) & 0.7 & 0.04 & 1.4 \\
Riboflavin (mg) & 0.6 & 0.03 & 1.5 \\
Niacin (mg) & 9.8 & 0.4 & 18 \\
Fe (mg) & 9.8 & 1.0 & 30 \\
Folate (mg) & 115 & 8 & 150 \\
\hline
\end{tabular}

are presented in Table 3 and Fig. 1. It may be observed that different sites responded differently during the period of the study. Femoral neck BMD showed a significant reduction at 6 months with partial recovery of BMD at 18 months. Changes in femoral neck bone area (BA) and BMC during this period were not statistically significant. Hip BMD reduced significantly at 6 months with gradual recovery to the baseline value by 18 months. Hip BA did not show significant changes, whereas hip BMC showed significant reduction at 6 months with return to baseline at 12 months itself. Forearm BMD showed reduction at 1 year with complete recovery by 18 months.

At lumbar spine, the loss of BMC and BMD at 6 months post-partum was not statistically significant and there was a significant improvement in these parameters at 12 and 18 months when compared with the baseline value. BA at lumbar spine did not change during this period.

There was a significant reduction in whole-body BMC at 6 months with a gradual recovery by 18 months. Surprisingly, maternal weight, lean mass and fat mass did not show significant change in spite of prolonged lactation. Growth faltering of infants was observed with progressive decline in weight for age and length for age $Z$ scores from 6 to 18 months.

\section{Regression analysis}

Regression models were constructed with per cent change in BMD at 6 months at each skeletal site as dependent variable, and baseline height, weight, lean mass, fat mass, age, parity, time of baseline measurements since delivery and duration of post-partum amenorrhoea as independent variables (Table 4). It was observed that baseline lean mass was the most important determinant of bone preservation or gain in BMD at femoral neck, hip as well as whole body, explaining 27,50 and $25 \%$ of variance, respectively, whereas baseline weight was the most important determinant of per cent gain in lumbar spine and fat mass was the most important determinant of per cent gain in forearm BMD explaining 18 and $19 \%$ of variance, respectively.

\section{Biochemical parameters}

The mean values of $\mathrm{Hb}$, serum protein, serum albumin, serum calcium and serum zinc were normal and did not change from baseline during lactation. Serum phosphorus values reduced when compared with the baseline, throughout the lactation period (from 44 (SE 1) $\mathrm{mg} / \mathrm{l}$ at baseline to 38 (SE 1) mg/l at 18 months, $P<0 \cdot 05)$. As expected, breast milk calcium values showed significant reduction from baseline at 12 and 18 months (from 236 (SE 14) mg/l at baseline to 148 (SE 8) mg/l at 18 months). Serum bone-specific alkaline phosphatase did not show a significant change during lactation when compared with the baseline value. On the other hand, serum tartrate-resistant acid phosphatase showed a significant reduction during lactation when compared with the baseline value (7.8 (SE 0.4) IU/1 at baseline to $5 \cdot 7$ (SE 0.5) IU/l at 18 months).

\section{Discussion}

This is the first study from India that investigates the lactation-related bone changes among undernourished 
Table 3. Changes in maternal bone, body composition and biochemical parameters and infant growth during 18 months post-partum (Mean values with their standard errors)

\begin{tabular}{|c|c|c|c|c|c|c|c|c|c|}
\hline & \multicolumn{2}{|c|}{ Baseline } & \multicolumn{2}{|c|}{6 months } & \multicolumn{2}{|c|}{12 months } & \multicolumn{2}{|c|}{18 months } & \multirow[b]{2}{*}{$P$ value } \\
\hline & Mean & SE & Mean & SE & Mean & SE & Mean & SE & \\
\hline \multicolumn{10}{|l|}{ Femoral neck ( $n$ 36) } \\
\hline BA & $4 \cdot 12$ & 0.10 & $4 \cdot 20$ & $0 \cdot 10$ & $4 \cdot 19$ & 0.11 & $4 \cdot 20$ & $0 \cdot 10$ & 0.535 \\
\hline BMC & 3.01 & 0.09 & $2 \cdot 91$ & 0.07 & 2.91 & 0.07 & 2.99 & 0.06 & 0.151 \\
\hline BMD & $0.73^{\mathrm{a}}$ & 0.01 & $0.70^{\mathrm{b}}$ & 0.01 & $0.70^{\mathrm{b}}$ & 0.01 & $0.72^{\mathrm{C}}$ & 0.01 & 0.000 \\
\hline \multicolumn{10}{|l|}{ Hip (n 36) } \\
\hline BA & $27 \cdot 02$ & 0.38 & $27 \cdot 15$ & 0.41 & $27 \cdot 25$ & 0.38 & $27 \cdot 38$ & 0.42 & 0.494 \\
\hline BMC & $20 \cdot 758^{a}$ & 0.40 & $20 \cdot 06^{b}$ & 0.39 & $20 \cdot 41^{\mathrm{a}}$ & 0.37 & $21.05^{\mathrm{a}}$ & 0.39 & 0.000 \\
\hline BMD & $0.77^{\mathrm{a}}$ & 0.01 & $0.74^{\mathrm{b}}$ & 0.01 & $0.75^{c}$ & 0.01 & $0.77^{\mathrm{a}}$ & 0.01 & 0.000 \\
\hline \multicolumn{10}{|l|}{ Lumbar spine (n 35) } \\
\hline $\mathrm{BA}$ & $47 \cdot 62$ & 0.71 & $47 \cdot 72$ & 0.74 & $49 \cdot 71$ & 1.63 & $49 \cdot 05$ & 0.76 & 0.105 \\
\hline BMC & $38.44^{\mathrm{a}}$ & 1.19 & $38.08^{a}$ & $1 \cdot 18$ & $41.93^{b}$ & 2.06 & $41.91^{b}$ & 1.18 & 0.000 \\
\hline BMD & $0.80^{\mathrm{a}}$ & 0.02 & $0.79^{\mathrm{a}}$ & 0.02 & $0.83^{\mathrm{b}}$ & 0.02 & $.0 .85^{\mathrm{c}}$ & 0.01 & 0.000 \\
\hline \multicolumn{10}{|l|}{ Forearm (n 28) } \\
\hline $\mathrm{BA}$ & 18.91 & 0.33 & 18.94 & 0.32 & $19 \cdot 06$ & 0.36 & $19 \cdot 06$ & 0.35 & 0.511 \\
\hline BMC & 9.88 & 0.19 & $9 \cdot 79$ & 0.21 & $9 \cdot 79$ & 0.22 & 9.96 & 0.19 & 0.392 \\
\hline BMD & $0.52^{\mathrm{a}}$ & 0.01 & $0.52^{\mathrm{a}}$ & 0.01 & $0.51^{\mathrm{b}}$ & 0.01 & $0.52^{\mathrm{a}}$ & 0.01 & 0.002 \\
\hline \multicolumn{10}{|l|}{ Whole body (n 32) } \\
\hline BA & 1584 & $15 \cdot 73$ & 1570 & 15.45 & 1578 & $15 \cdot 61$ & 1585 & 15.41 & 0.085 \\
\hline BMC & $1589^{a}$ & $24 \cdot 65$ & $1567^{\mathrm{b}}$ & 25.91 & $1580^{\mathrm{ab}}$ & 26.99 & $1602^{\mathrm{a}}$ & 24.59 & 0.002 \\
\hline BMD & $1.00^{\mathrm{ab}}$ & 0.01 & $1.00^{\mathrm{a}}$ & 0.01 & $1.00^{\mathrm{a}}$ & 0.01 & $1 \cdot 01^{\mathrm{b}}$ & 0.01 & 0.027 \\
\hline Lean mass & 30.04 & 0.43 & 29.86 & 0.42 & $29 \cdot 78$ & 0.39 & 30.00 & 0.41 & 0.491 \\
\hline Fat mass & $12 \cdot 73$ & 0.62 & $12 \cdot 90$ & 0.70 & $12 \cdot 21$ & 0.76 & $12 \cdot 15$ & 0.74 & 0.112 \\
\hline Percentage fat & $28 \cdot 3^{a b}$ & 0.90 & $28 \cdot 5^{\mathrm{a}}$ & 1.01 & $27 \cdot 3^{\mathrm{b}}$ & $1 \cdot 10$ & $27 \cdot 1^{\mathrm{b}}$ & 1.07 & 0.025 \\
\hline Weight & $45 \cdot 8$ & 0.95 & $45 \cdot 4$ & 1.01 & $45 \cdot 0$ & 1.07 & 44.6 & $1 \cdot 14$ & 0.180 \\
\hline Weight of infant & 3.1 & 0.07 & 6.5 & 0.13 & 7.9 & 0.15 & $8 \cdot 6$ & 0.17 & \\
\hline WAZ & $-0.9^{a}$ & 0.13 & $-0.83^{\mathrm{a}}$ & 0.15 & $-1.58^{b}$ & 0.15 & $-1.94^{c}$ & 0.15 & 0.020 \\
\hline Length of infant & 50.6 & 0.36 & 65.4 & 0.53 & $72 \cdot 8$ & 0.50 & $77 \cdot 4$ & 0.58 & \\
\hline LAZ & $0.33^{\mathrm{a}}$ & 0.17 & $-0.20^{\mathrm{ab}}$ & 0.20 & $-0.54^{b}$ & 0.19 & $-1 \cdot 15^{c}$ & 0.21 & 0.027 \\
\hline BSAP & $29 \cdot 3$ & $2 \cdot 8$ & $36 \cdot 4$ & 4.4 & $25 \cdot 8$ & 3.5 & 22.9 & $3 \cdot 2$ & 0.065 \\
\hline TRAP & $7 \cdot 8^{a}$ & 0.4 & $4 \cdot 6^{\mathrm{b}}$ & 0.4 & $4.9^{\mathrm{b}}$ & 0.5 & $5 \cdot 7^{\mathrm{b}}$ & 0.5 & 0.000 \\
\hline
\end{tabular}

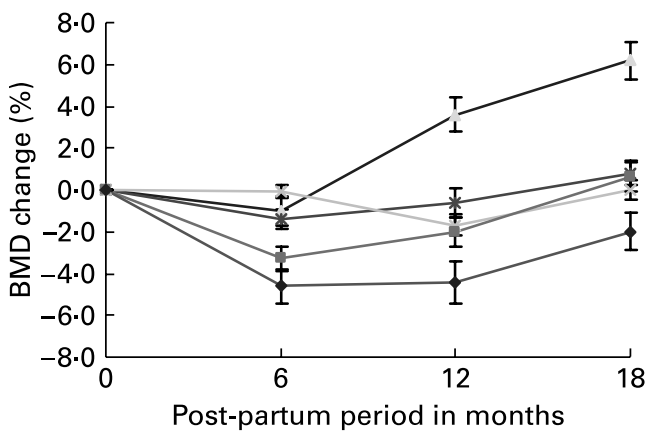

Fig. 1. Bone mineral density (BMD) changes (per cent) at different skeletal sites and whole body during 18 months post-partum; $n 36(\rightarrow-$, femoral neck; $-\rightarrow$, hip), 35 (--, lumbar spine), 32 ( $\rightarrow$, whole body) and $28(\rightarrow$, forearm). values are means, with standard errors represented by vertical bars. There was a significant reduction in BMD at hip as well as the whole-body bone mineral content (WB-BMC) at 6 months with complete recovery by 18 months. Femoral neck (FN) BMD reduced significantly at 6 months with a partial recovery at 18 months. Forearm BMD reduced significantly at 12 months with recovery at 18 months. Loss of lumbar spine BMD at 6 months was not statistically significant and the BMD increased significantly at 12 and 18 months when compared with the baseline. women consuming diets with gross inadequacy of all the important nutrients, especially calcium.

The overall nutritional status of the study women as indicated by their height, weight, BMI and dietary intakes was comparable to that reported by National Nutrition Monitoring Bureau surveys, which are large-scale nutrition surveys of the low socio-economic population from India ${ }^{(13)}$. The mean birth weight of their infants also corresponded to the mean birth weight reported for the low-income group population from India (Table 1).

The women breast-fed their babies throughout the study period with inadequate complementary feeds. Early introduction of animal milk in small quantities may have reduced the period of lactation amenorrhoea to 7 months in contrast to the earlier studies reported from the same population where it was 11 months ${ }^{(22)}$. This practice of early introduction of animal milk to infants may not have affected growth in the first 6 months as indicated by their weight for age $Z$ scores at baseline and at 6 months, but it may have hastened the return of menstruation. The effect of poor complementary feeding on growth of infants was apparent after 6 months of age.

The mean values of bone parameters were much lower than those reported for Western women of similar age group, indicating poor bone health of the study women ${ }^{(7,12)}$. When the 
Table 4. Regression models of per cent change in bone mineral density bone mineral density (BMD) at different skeletal sites at 6 months with height, weight, age, parity, duration of post-partum amenorrhoea, baseline fat mass and lean mass, and time of baseline measurements since delivery as independent variables

\begin{tabular}{|c|c|c|c|c|}
\hline Dependent variable & Significant variables & $R$ & $R^{2}$ & $P$ value \\
\hline Percentage change in FN BMD at 6 months ( $n 36)$ & Lean mass & 0.52 & $0 \cdot 27$ & 0.002 \\
\hline Percentage change in hip BMD at 6 months $(n 36)$ & Lean mass & 0.71 & 0.50 & 0.000 \\
\hline Percentage change in lumbar spine BMD at 6 months $(n 35)$ & Weight & 0.42 & $0 \cdot 18$ & 0.013 \\
\hline Percentage change in whole body BMD at 6 months ( $n$ 32) & Lean mass & 0.50 & $0 \cdot 25$ & 0.003 \\
\hline Percentage change in forearm BMD at 6 months $(n 28)$ & Fat mass & 0.44 & 0.19 & 0.01 \\
\hline
\end{tabular}

FN, femoral neck.

changes in bone parameters were studied at 6, 12 and 18 months of lactation, it was observed that the women suffered from a significant loss of BMD at femoral neck (4.6\% loss) at 6 months. There were no signs of recovery at 12 months, with only a partial recovery at 18 months (Table 3; Fig. 1). This finding is not in agreement with similar studies reported from the west. Most of the Western studies reported restoration of femoral neck BMD by 18 months post-partum ${ }^{(23,24)}$. On the other hand, loss of BMD at hip showed complete recovery at 18 months in the present study.

The changes in BA and BMC at different skeletal sites showed an interesting pattern. At the spine and total hip, changes in BMC appear to determine the changes in BMD. However, at the femoral neck and forearm, changes in BMD appear to be the result of both an increase in BA and a decrease in $\mathrm{BMC}$, though these changes in $\mathrm{BA}$ and $\mathrm{BMC}$ are not significant.

Surprisingly, at lumbar spine, no significant reduction in BMD was observed at 6 months, despite the calcium drain in breast milk. This is in contrast to virtually all the studies reported in lactating women as none of these studies have shown the preservation of lumbar spine BMD (LS-BMD) at 6 months of lactation ${ }^{(4-9)}$. There was a wide variation in per cent change in LS-BMD, with some women losing up to $6.7 \%$ of $\mathrm{BMD}$ and some women gaining up to $16 \%$ of BMD at 6 months post-partum. Studies from the west indicate that LS-BMD loss occurred even among women having high dietary intake of calcium and shorter duration of lactation ${ }^{(5-7)}$. In the present study, LS-BMD showed a significant increase at 12 and 18 months when compared with the baseline value (3.6 and $6.3 \%$, respectively). Biochemical marker of bone resorption (serum tartrate-resistant acid phosphatase) showed a significant reduction at 6 months, whereas bone-specific alkaline phosphatase, which is a marker of bone formation, did not show a significant change when compared with the baseline. It was not possible to measure vitamin D and parathyroid hormone levels in the study participants. Vitamin D insufficiency is associated with secondary hyperparathyroidism and bone loss mainly from cortical sites, similar to the changes observed in the present study ${ }^{(25-27)}$. However, studies investigating biochemical correlates of BMD changes during lactation have shown that calcitrophic hormones such as parathyroid hormone, 25-hydroxy vitamin $\mathrm{D}$ and 1,25dihydroxy vitamin $\mathrm{D}$ do not play a central role in calcium mobilisation associated with bone loss of lactation ${ }^{(28,29)}$.

The women in the present study were younger when compared with the women from the Western studies. It may be speculated that the absence of LS-BMD loss in the present study may be because of an attempt to increase the BMD for PBM acquisition at this young age. But this finding is in contrast to a number of studies in adolescent mothers from the west who had a loss of BMD at the hip and spine ${ }^{(30,31)}$, despite their young age. Western studies in well-nourished adolescents have showed that they attained PBM shortly after puberty $^{(32,33)}$ It needs to be explored whether undernutrition delays the age at PBM consolidation in the Indian context. It is known that undernutrition delays the attainment of adult height to as late as 20 years ${ }^{(34)}$. Studies investigating the age at PBM development among undernourished populations in India are not available. Cross-sectional data from this Institute suggest that PBM may not be attained till 25-30 years of age even among Indian population from high income group with calcium intakes of about $800 \mathrm{mg} / \mathrm{d}^{(35)}$. Different patterns of change at different skeletal sites may be linked to the differences in the age at which PBM is attained at those sites. Studies in Caucasian youth indicated that peak volumetric BMD at femoral neck was reached much earlier than lumbar spine in both men and women ${ }^{(36)}$. A longitudinal study from Sweden also corroborated these findings ${ }^{(37)}$.

It is remarkable that despite poor dietary calcium intakes of about $450 \mathrm{mg} / \mathrm{d}$ and breast milk calcium losses of about $200 \mathrm{mg} / \mathrm{d}$, these women could resist bone loss at lumbar spine at 6 months and could increase LS-BMD by more than $6 \%$ at 18 months. Lack of significant decline in BMD at lumbar spine may reflect the delayed baseline measurements (within 1 month post-partum, not within 0.5 month as used by other studies) and hence, some decrease in BMD may have been missed.

Changes in forearm BMD, whole-body BMC and whole body BMD were transient and showed complete recovery at 18 months. It may be speculated that hormonal interplay and metabolic adjustments in young undernourished women may lead to an internal reorganisation of mineral in such a way that lumbar spine is privileged.

Increased bone turnover in the hypo-oestrogenic phase of post-partum amenorrhoea is known to be qualitatively similar to menopause. But unlike menopause, when metabolically active trabecular bone at lumbar spine shows rapid loss of bone density, lactation was associated with significant increase in lumbar spine BMD in the present study.

Surprisingly, in spite of prolonged lactation and poor dietary intakes of almost all the nutrients including energy, these women did not lose weight, lean mass or fat mass. Data collected from the women showed that they probably conserved energy by opting out of the labour force for a period of $1-1.5$ years. 
Regression analysis indicates that maternal weight and specifically lean body mass explain major variation in BMD changes at various skeletal sites among undernourished women.

A higher weight may have a positive effect on bone density by either bone-loading effect or it may be an indicator of higher nutrient reserves available for utilisation during the nutritionally demanding phase of lactation. Western studies in well-nourished subjects have not reported the relationship of maternal weight to the lactation-related bone changes. It may be speculated that among undernourished populations maintaining precarious nutrient balance, availability of nutrient reserves becomes important for resisting lactation-related bone changes.

Earlier studies from this Institute demonstrated that in undernourished populations, nutritional status as indicated by body weights and BMI is a major determinant of bone health $^{(15)}$. The present study demonstrates that even during dynamic phase of lactation characterised by hormonal changes and increased calcium demand, nutritional status of women, as indicated by weight, lean mass and fat mass, continuing to be a major determinant of bone changes.

It is interesting to note that in the present study, lean body mass was a major determinant of a preservation of BMD at femoral neck, hip and whole-body BMC. Lean body mass is known to be a major determinant of development and maintenance of BMD at the hip region ${ }^{(38)}$. Muscle mass is the major constituent of the lean body mass and the positive effect of lean mass on BMD is thought to be mediated by its bone-loading effect as well as osteogenic stimulation by muscle force. It is remarkable that these functions of lean mass continue to be important for preservation of bone density at femoral neck and hip during lactation. Other studies have not looked for these relationships. It would be important to see the effect of different lean body mass values at a given BMI on lactation-related bone changes.

The present study thus provides important information regarding the lactation-related bone changes in young Indian women from the low socio-economic group. The study is unique with subjects having grossly inadequate intakes of all the nutrients including calcium, prolonged breastfeeding with little complementary foods and follow-up till 18 months post-partum. The results indicate that at the time of building maternal PBM, maternal weight, lean body mass and fat mass are important determinants of bone preservation during lactation. The study raises important questions regarding calcium balance in lactating women during the growth phase and stimulates further research to explore the relevance of nutrition to the bone changes during lactation.

\section{Acknowledgements}

The present study was funded by the Indian Council of Medical Research. The sponsor of the study had no role in study design, data collection, data analysis, data interpretation or writing of this manuscript. The authors declare that they have no conflict of interest. B. K. and V. S. contributed to the study design, subject enrolment, interpretation of the results and the manuscript preparation. N. B. carried out statistical analysis of the data. P. A. K. was responsible for the biochemical estimations, K. U. R. for the bone measurements by dual-energy X-ray absorptiometry and A. C. O. for the estimations of dietary intakes. All the authors reviewed and approved the final version of the manuscript. The authors would like to thank the directors of the National Institute of Nutrition, Hyderabad, India, who provided the facilities and support system to carry out this work. They are grateful to Ms B. Prema Kumai, Ms K. Sundaramma and $\mathrm{Mr}$ R. Sambasiva Rao for their help during the course of the study. The present study would not have been possible without the unstinting cooperation of the women who were subjects of the study and the authors are grateful to them.

\section{References}

1. Vargas Zapata CL, Donangelo CM \& Woodhouse LR (2004) Calcium homeostasis during pregnancy and lactation in Brazilian women with low calcium intakes: a longitudinal study. Am J Clin Nutr 80, 417-422.

2. Ritchie LD, Fung EB, Halloran BP, et al. (1998) A longitudinal study of calcium homeostasis during human pregnancy and lactation and after resumption of menses. Am J Clin Nutr 67, 693-701.

3. Holmberg-Marttila D, Leino A \& Sievanen H (2003) Bone turnover markers during lactation, postpartum amenorrhea and resumption of menses. Osteoporos Int 14, 103-109.

4. Chan SM, Nelson EA, Leung SS, et al. (2005) Bone mineral density and calcium metabolism of Hong Kong Chinese postpartum women - a 1-y longitudinal study. Eur J Clin Nutr 59, $868-876$.

5. Karlsson C, Obrant KJ \& Karlsson M (2001) Pregnancy and lactation confer reversible bone loss in humans. Osteoporos Int 12, 828-834.

6. Affinito P, Tommaselli GA, di Carlo C, et al. (1996) Changes in bone mineral density and calcium metabolism in breastfeeding women: a one year follow-up study. J Clin Endocrinol Metab 81, 2314-2318.

7. More C, Bettembuk P, Bhattoa HP, et al. (2001) The effects of pregnancy and lactation on bone mineral density. Osteoporos Int 12, 732-737.

8. Bezerra FF, Mendonca LM, Lobato EC, et al. (2004) Bone mass is recovered from lactation to postweaning in adolescent mothers with low calcium intakes. Am J Clin Nutr 80, $1322-1326$.

9. Krebs NF, Reidinger CJ, Robertson AD, et al. (1997) Bone mineral density changes during lactation: maternal, dietary, and biochemical correlates. Am J Clin Nutr 65, 1738-1746.

10. O'Brien KO, Donangelo CM, Zapata CL, et al. (2006) Bone calcium turnover during pregnancy and lactation in women with low calcium diets is associated with calcium intake and circulating insulin-like growth factor 1 concentrations. Am J Clin Nutr 83, 317-323.

11. Cross NA, Hillman LS, Allen SH, et al. (1995) Calcium homeostasis and bone metabolism during pregnancy, lactation, and postweaning: a longitudinal study. Am J Clin Nutr 61, 514-523.

12. Kalkwarf HJ, Specker BL, Bianchi DC, et al. (1997) The effect of calcium supplementation on bone density during lactation and after weaning. $N$ Engl J Med 337, 523-528.

13. National Nutrition Monitoring Bureau (2002) Diet and Nutritional Status of Rural Population. Hyderabad: National Institute of Nutrition.

14. Shatrugna V (1998) Osteoporosis in the Asian region: newer questions. In Diet, Nutrition and Chronic Diseases, pp. 81-83 [P Shetty and C Gopalan, editors]. London: Smith-Gordon.

15. Shatrugna V, Kulkarni B, Kumar PA, et al. (2005) Bone status of Indian women from a low income group and its relationship to the nutritional status. Osteoporos Int 16, 1827-1835. 
16. Gupta AK, Samuel KC, Kurian PM, et al. (1967) Preliminary study of the incidence and aetiology of femoral neck fractures in Indians. Indian J Med Res 55, 1341-1348.

17. Gustafsson JE (1976) Improved specificity of serum albumin determination and estimation of 'acute phase reactants' by use of the bromcresol green reaction. Clin Chem 22, 616-622.

18. Schiele F, Arthur Y, Floc'h AY, et al. (1988) Total, tartrate resistant and tartrate-inhibited acid phosphatases in serum: biological variations and reference limits. Clin Chem 34, 685-690.

19. Cadeau BJ \& Malkin A (1973) A relative heat stability test for the identification of serum alkaline phosphatase isoenzymes. Clin Chim Acta 45, 235-242.

20. National Institute of Nutrition (1989) Nutritive Value of Indian Foods. Hyderabad: Indian Council of Medical Research.

21. Indian Council of Medical Research (1989) Nutrient Requirement And Recommended Dietary Allowances for Indians. A Report of the Expert Group of the ICMR. Hyderabad: NIN.

22. Prema K, Naidu AN, Neelakumari S, et al. (1981) Nutritionfertility interaction in lactating women of low income groups. Br J Nutr 45, 461-467.

23. Laskey MA \& Prentice A (1999) Bone mineral changes during and after lactation. Obstet Gynecol 94, 608-615.

24. Sowers M, Corton G, Shapiro B, et al. (1993) Changes in bone density with lactation. J Am Med Assoc 269, 3130-3135.

25. Sigurdssan G, Franzsan L, Steingrimsdottir L, et al. (2000) The association between parathyroid hormone, vitamin $\mathrm{D}$ and bone mineral density in 70 year old Icelandic women. Osteoporos Int. 11, 1031-1035.

26. Bhattoa HP, Bettembuk P, Ganacharya S, et al. (2004) Prevalence and seasonal variation of hypovitaminosis D and its relationship to bone metabolism in community dwelling postmenopausal Hungarian women. Osteoporos Int 15, 447-451.

27. Duan Y, Deluca V \& Seeman E (1999) Parathyroid hormone deficiency and excess: similar effects on trabecular bone but differing effects on cortical bone. J Clin Endocrinol Metab 84, $718-722$.
28. DeSantiago S, Alonso L, Halhali A, et al. (2002) Negative calcium balance during lactation in rural Mexican women. $\mathrm{Am}$ $J$ Clin Nutr 76, 845-851.

29. Sowers M, Zhang D, Hollis BW, et al. (1998) Role of calciotrophic hormones in calcium mobilization of lactation. Am J Clin Nutr 67, 284-291.

30. Chan GM, Ronald N, Slater P, et al. (1982) Decreased bone mineral status in lactating adolescent mothers. J Pediatr 101, $767-770$.

31. Sowers MF, Scholl T \& Harris L (2000) Bone loss in adolescent and adult pregnant women. Obstet Gynecol 96, 89-93.

32. Sabatier JP, Guaydier-Souquieres G, Laroche D, et al. (1996) Bone mineral acquisition during adolescence and early adulthood: a study in 574 healthy females 10-24 years of age. Osteoporos Int 6, 141-148.

33. Van Coeverden SC, De Ridder CM, Roos JC, et al. (2001) Pubertal maturation characteristics and the rate of bone mass development longitudinally toward menarche. J Bone Miner Res 16, 774-781.

34. Satyanarayana K, Radhaiah G, Mohan KR, et al. (1989) The adolescent growth spurt of height among rural Indian boys in relation to childhood nutrition background: an 18 year longitudinal study. Ann Hum Biol 16, 289-300.

35. National Institute of Nutrition (2007) Annual Report. Hyderabad: National Institute of Nutrition.

36. Henry YM, Fatayerji D \& Eastell R (2004) Attainment of peak bone mass at the lumbar spine, femoral neck and radius in men and women: relative contributions of bone size and volumetric bone mineral density. Osteopro Int 15, 263-273.

37. Sundberg M, Gardsell P, Johnell O, et al. (2003) Pubertal bone growth in the femoral neck is predominantly characterized by increased bone size and not by increased bone density - a 4-year longitudinal study. Osteoporos Int 14, 548-558.

38. Vicente-Rodriguez G, Ara I, Perez-Gomez J, et al. (2005) Muscular development and physical activity as major determinants of femoral bone mass acquisition during growth. Br J Sports Med 39, 611-616. 